\title{
Interface Modification of DNTT-Based Organic Field Effect Transistors Using Boronic Acid Derivatives
}

Tuğbahan Yılmaz Alıç ${ }^{1,2}$, Abduleziz Ablat ${ }^{2}$, Adrica Kyndiah ${ }^{2}$, Yohann Nicolas ${ }^{3}$, Mustafa $\mathrm{Can}^{4}$, Mahmut Kuş ${ }^{5 *}$ and Mamatimin Abbas ${ }^{2 *}$

${ }^{1}$ Selcuk University, Departmant of Physics, Konya/Turkey

${ }^{2}$ Université de Bordeaux, Laboratoire IMS, UMR CNRS 5218, ENSCBP, Pessac, France

${ }^{3}$ C2M - ISM Université de Bordeaux 351 cours de la libration 33405 Talence, France

${ }^{4}$ Katip Celebi University, Departmant of Engineering Sciences, İmir, Turkey

${ }^{5}$ Gebze Technical University, Institute of Energy Technologies, Gebze, Kocaeli, Turkey

\begin{abstract}
Dielectric/semiconductor interface in organic field effect transistors (OFETs) is critical in their performances. Modification of this interface with functional molecules provides a wide range of possibilities for their applications as sensors. In this work, boronic acid molecules were used to modify $\mathrm{SiO}_{2}$ dielectric surface in dinaphtho[2,3-b:2',3'-f]thieno[3,2-b]thiophene (DNTT) based OFETs. The device parameters, notably threshold voltage was significantly improved. Dielectric/semiconductor interface was analyzed by various measurement techniques, such as contact angle and atomic force microscopy. Our work puts in evidence that easily functionable boronic acid derivatives improve the device performances of OFETs, laying foundation for further studies of such interface modified OFETs towards sensing applications.
\end{abstract}

Keywords: Boronic acid derivatives, dielectric/semiconductor interface, organic field effect transistor

\section{Introduction}

Organic semiconductors are of great interest for research community potentially being the main components of future electronics. They have several advantages over conventional semiconductors as they can be easily processed at low cost. A wide range of materials are available thanks to the rational chemical design and synthesis. Devices based on them can have quite appealing characteristics such as being flexible, printable, light weight and large area. ${ }^{1}$ Among them, organic field effect transistor (OFET) is an essential building block of various electronic circuits. ${ }^{2}$ In an OFET, charge-transport occurs mainly at the semiconductor/dielectric interface. Any modifications at this interface can have significant impact on OFET device performance, which renders the potential of OFET to be used in sensing applications.

Boronic acids are an important class of compounds in chemistry and medicine. They can be easily handled, thus becoming excellent choice for synthesis intermediates. ${ }^{3,4}$ Phenylboronic 
acid derivatives have been extensively studied as sensors for sugars, as they are well-known to form complexes with diol of saccharides.$^{5-8}$ Extended gate approach where gate electrodes of field effect transistors (FET) are functionalized with boronic acid molecules has been proven to detect efficiently various saccharides and also biomolecule. ${ }^{9-13}$ Comparing to gate modification, current modulation of FETs is in principle much more sensitive to dielectric/semiconductor interface modification, which is the main medium for charge transport. Therefore, incorporation of boronic acid molecules in dielectric surface of an FET can be a viable approach for further enhancing the sensitivity, which has not been investigated so far. Here, the primary question raised would be how much the original performance of FETs get affected by such functionalization. In this work, we modified the dielectric surface of $\mathrm{SiO}_{2}$ with fluorinated phenylboronic acid molecules in an organic field effect transistor (OFET) with the aim to investigate their effect on the device performance. We observed that these selfassembled molecules improve the device performance parameters of our OFETs, notably the threshold voltage, revealing their potential to act as both passivation and sensing layer.

\section{Experimental}

Highly n-doped silicon substrates with thermally grown $\mathrm{SiO}_{2}(200 \mathrm{~nm})$ were used respectively as gate and dielectric in a bottom gate, top contact device configuration, as shown in Figure 1. As an active layer, thienoacene-based organic semiconductor dinaphtho[2,3-b:2',3'f]thieno[3,2-b]-thiophene (DNTT) was chosen for its excellent hole mobility and air stability. ${ }^{14-}$ 17 (4-fluorophenyl) boronic acid (1-F), (3,5-fluorophenyl) boronic acid (2-F) and (3,4,5trifluorophenyl) boronic acid (3-F) molecules used for dielectric surface treatments are also shown. They were synthesized using 1-Bromo-4-fluorobenzene, 1-bromo-3,5-difluorobenzene and 5-bromo-1,2,3-trifluorobenzene source materials by subsequently adding dropwise nbutyllithium and trimethylborate at $-80^{\circ} \mathrm{C}$ in tetrahydrofuran (THF). ${ }^{1} \mathrm{H}-\mathrm{NMR}$ data show that 2-F contains slightly higher impurities than the others (see supporting information). They were dissolved in THF at $1 \mathrm{mM}$ concentration. $\mathrm{SiO}_{2}$ substrates were then immersed in it for $24 \mathrm{~h}$ for self-assembly at room temperature. After rinsing and drying the substrates, they were transferred to the nitrogen glove box. $20 \mathrm{~nm}$ of DNTT organic semiconducting layer was evaporated thermally in a vacuum at a pressure of about $10^{-6}$ mbar. The deposition rate $(0.1$ $\AA / s$ ) and the film thickness were monitored using a quartz crystal microbalance. $\mathrm{WO}_{3} / \mathrm{Au}$ source and drain contacts were deposited by thermal evaporation (thickness $10 \mathrm{~nm} / 60 \mathrm{~nm}$ ) through a shadow mask to finalize a device with a channel length (L) of $50 \mu \mathrm{m}$ and a channel width (W) of $1 \mathrm{~mm} .{ }^{17}$ 


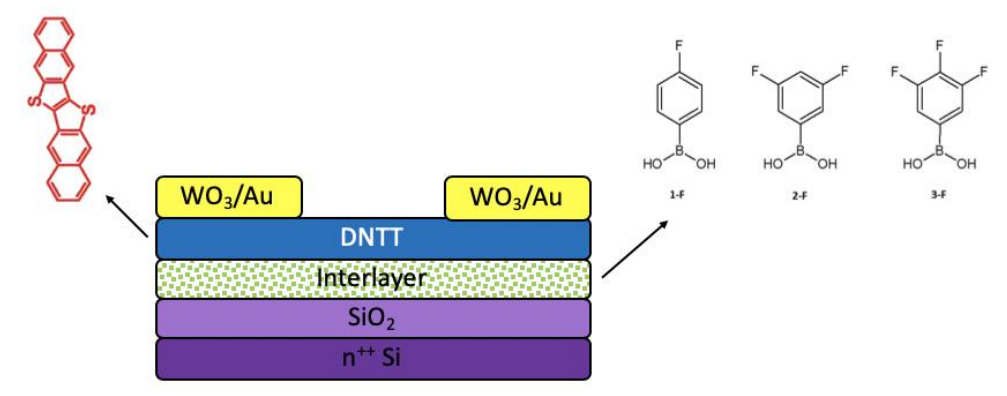

Figure 1. Bottom gate, top contact OFET device structure; chemical structure of DNTT active layer; chemical structures of fluorinated phenylboronic acid molecules.

The surface analyses of Self-assembled-monolayers (SAMs) treated and non-treated substrates were carried out by contact angle goniometer (DSA 100 KRÜSS) and atomic force microscope (AFM) (NT-MDT AFM NTEGRA Solaris) in tapping mode in ambient air at room temperature. Current-voltage $(I-V)$ measurements on the OFETs were carried out in nitrogen glovebox using a micromanipulator probe station and a 4200 Keithley semiconductor parameter analyzer.

\section{Results and Discussions}

In order to investigate the surface properties of the $\mathrm{SiO}_{2}$ after the treatment by phenylboronic acid molecules, we carried out atomic force microscopy (AFM) analysis. Figure 2 displays the height images of various surfaces captured in tapping mode. We see that the grafting is quasi-total on the oxide surface despite some aggregations. Root mean square roughness changed from $0.2 \mathrm{~nm}$ for the bare oxide to $0.3 \mathrm{~nm}, 0.45 \mathrm{~nm}$ and $0.25 \mathrm{~nm}$ for $1-\mathrm{F}, 2-$ $\mathrm{F}$ and 3-F treated surfaces, respectively. Although the same processing conditions were applied for all these layers, higher ratio of aggregation in the case of 2-F sample is probably due to the lower purity of the source material.
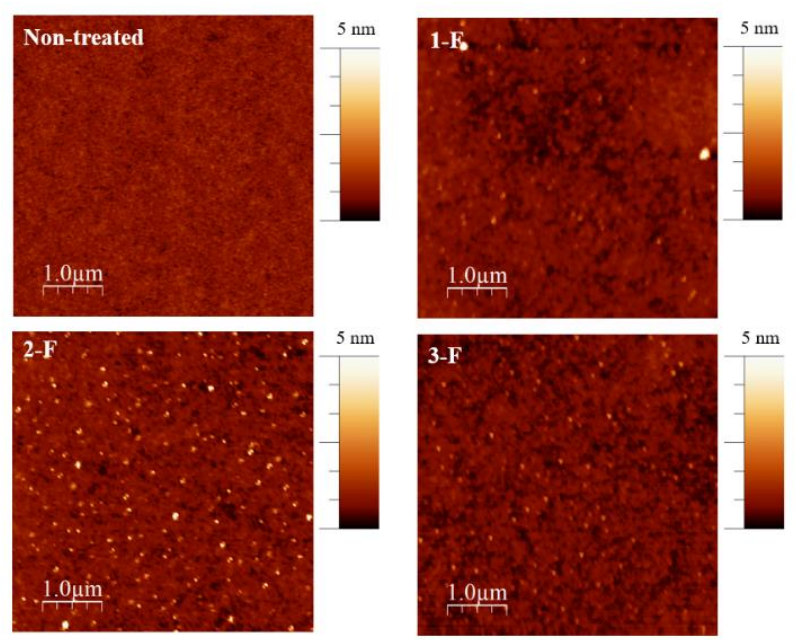

Figure 2. Atomic force microscopy (AFM) height images of the surfaces of bare $\mathrm{SiO}_{2}$ (non-treated) and treated with 1-F, 2-F and 3-F phenylboronic acid molecules. 
SAMs based on trichlorosilane or phosphoric acids anchor groups have shown dramatic effect on the charge carrier mobilities in OFET devices, mainly by tuning the surface energy of the oxides. ${ }^{18-20}$ To investigate how phenylboronic acids act on $\mathrm{SiO}_{2}$ surface, water contact angle measurements have been carried out, which we present in Figure 3. Calculated surface energies for all the surfaces are essentially the same being around $58 \mathrm{mN} / \mathrm{m}$. This value for bare $\mathrm{SiO}_{2}$ corresponds to those reported in literatures. $^{21}$
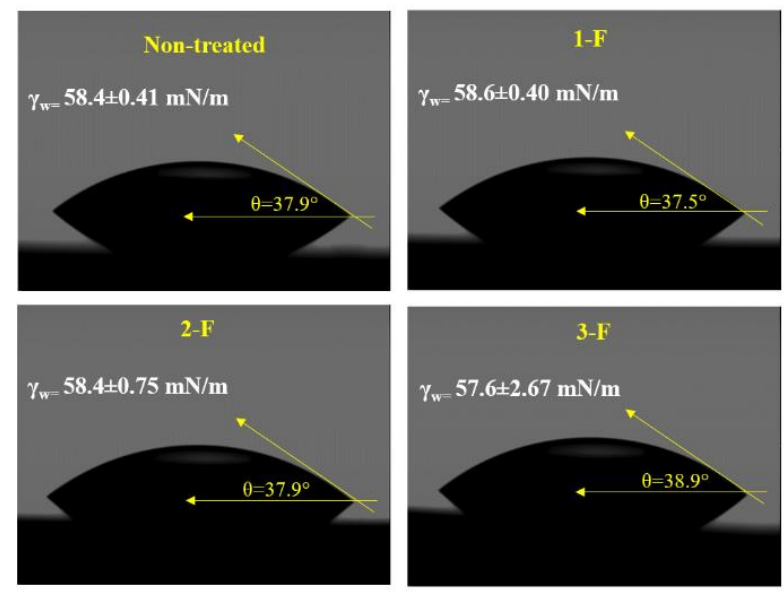

Figure 3. Water contact angles and calculated surface energies: the surfaces of bare $\mathrm{SiO}_{2}$ (non-treated) and treated with 1-F, 2-F and 3-F phenylboronic acid molecules.

Figure 4 shows the typical transfer and output curves of OFET devices: non-treated (on bare silicon oxide) and treated with three phenylboronic acid molecules. All the devices in general exhibit excellent electrical characteristics. Current modulations reach more than $10^{6}$ in gate voltage sweeps. Proper linear and saturation behaviors can be observed in drain voltage sweeps. Notable difference between the non-treated and treated devices is in the shape of the transfer curves with forward and backward scans. A characteristics triangular shape at low gate voltage region in the non-treated device can be attributed to the presence of deep traps at the interface. ${ }^{22}$ Such characteristics are not observed in any of the devices those treated with phenylboronic acid molecules, an indication of passivated surface by these molecules. All the devices show a slight hysteresis though, probably coming from the impurities or structural defects in the active layer. 

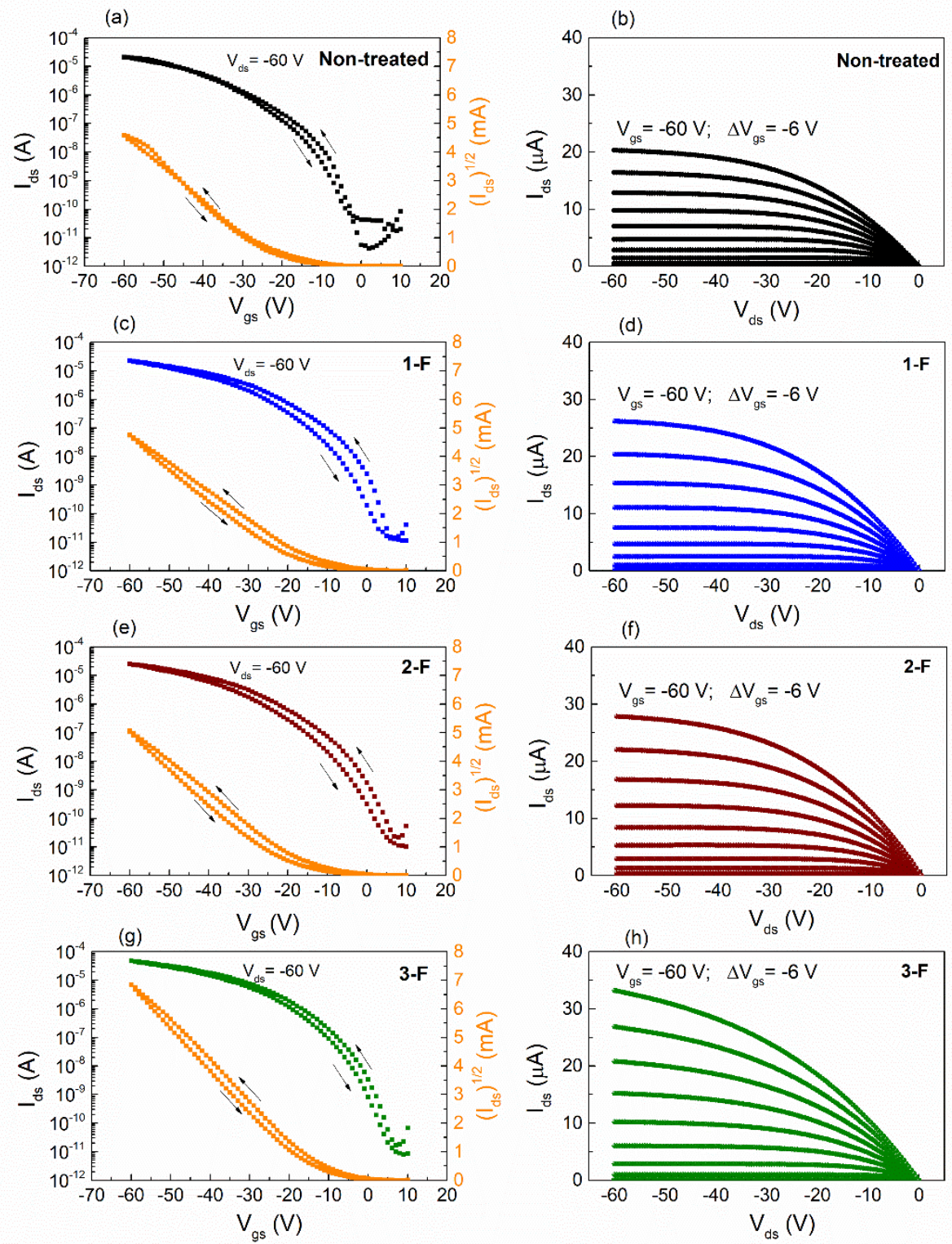

Figure 4. Transfer (a) and output (b) characteristics of the devices on bare $\mathrm{SiO}_{2}$ (non-treated); those of the devices treated with phenylboronic acid molecules: 1-F (c) and (d); $2-F(e)$ and (f); 3-F (g) and (h).

Linear and saturation mobilities were calculated from the following formula:

$\mu_{\text {lin }}=\frac{L}{C_{i} W V_{d s}}\left(\frac{\partial I_{d s}}{\partial V_{g s}}\right) ; \mu_{s a t}=\frac{2 L}{C_{i} W}\left(\frac{\partial \sqrt{I_{d s}}}{\partial V_{g s}}\right)^{2}$

where $C_{i}$ is the dielectric capacitance, $W$ is the channel width and $L$ is the channel length. The maximum values were taken from the gate voltage dependent mobility curves, as shown in Figure 5. Mobilities either in linear or saturation regimes of the non-treated and phenylboronic acid grafted devices yield in general similar mobilities. Even though 3-F gives a slightly higher mobilities, the variation is within the uncertainty range. Slight increase in the surface roughness coupled with aggregates, in principle can have a negative effect on the mobilities of the devices, 
which could be compensated by the positive effect of the decrease in interface trap densities (as we discussed earlier), thus resulting in similar final mobilities for all the devices. It should be noted however that for each device, the difference between linear and saturation mobilities is relatively small, an indication of optimal charge injection from the electrode to the active layer. The mobility of the devices on the bare $\mathrm{SiO}_{2}$ is relatively low, similar to that of the previous report on non-treated $\mathrm{SiO}_{2}$ surface. ${ }^{23}$


Figure 5. Gate voltage dependant mobilities of Non-treated, 1-F, 2-F and 3-F treated OFET devices in saturation (left panel) and linear (right panel) regimes.

In Figure 6 we present the comparison of OFET device performance parameters. At least ten devices were measured. Threshold voltage $\left(\mathrm{V}_{\text {th }}\right)$ is another important parameter in OFET device performance. We derived it from the square root of the drain current by extending the linear part to the zero current. We also define hysteresis $\left(\Delta \mathrm{V}_{\text {th }}\right)$ as the change in $\mathrm{V}_{\text {th }}$ of the forward and backward scanned curves. Here, we observe notable improvement in the performance of the devices with phenylboronic acid treatment. Threshold voltage decreased from around -20 $\mathrm{V}$ in non-treated device to about $-8 \mathrm{~V}$ in all the three treated devices. $\Delta \mathrm{V}_{\text {th }}$ also reduced from around $10 \mathrm{~V}$ to about $5 \mathrm{~V}$. This is a strong evidence that phenylboronic acids efficiently passivate the oxide surface, by saturating charge trapping species. One can also note that the turn-on voltage also shifted about $5 \mathrm{~V}$ following phenylboronic acids treatment, suggesting a formation of interface dipole at the phenylboronic acid/ $\mathrm{SiO}_{2}$ interface, which also contributed to the reduction of $\mathrm{V}_{\text {th }}$. Subthreshold slope (SS) is directly correlated to the interface trap densities. Indeed we obtained SS of around $5 \mathrm{~V} / \mathrm{dec}$ for treated devices instead of $7.5 \mathrm{~V} / \mathrm{dec}$ for the non-treated device, again confirming the reduction of trap densities at the interface following phenylboronic acid treatment. We did not observe notable differences in current on/off ratio among the devices, which are consistent with the mobility values. 

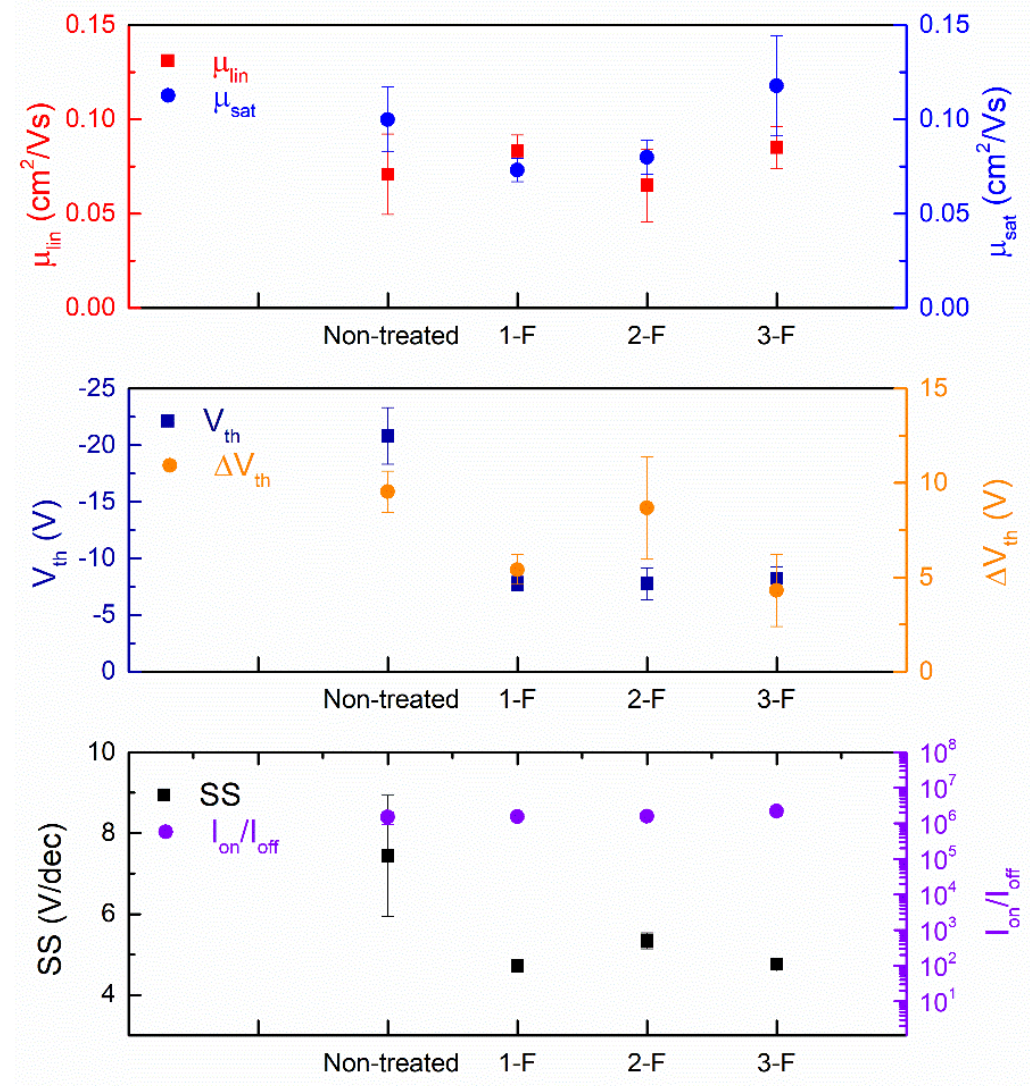

Figure 6. Device performance parameters of OFETs with non-treated and phenylboronic acid grafted $\mathrm{SiO}_{2}$ dielectric: linear mobility $\left(\mu_{\text {lin }}\right)$, saturation mobility $\left(\mu_{\text {sat }}\right)$, threshold voltage $\left(V_{t h}\right)$, hysteresis $\left(\Delta V_{t h}\right)$, subthreshold slope $(S S)$ and current on/off ratio $\left(I_{o n} / l_{\text {off }}\right)$.

Device performance parameters are summarized in Table 1.

Table 1. Summary of device performance parameters of OFETs with non-treated and phenylboronic acids grafted $\mathrm{SiO}_{2}$ dielectric

\begin{tabular}{|c|c|c|c|c|c|c|}
\hline & $\begin{array}{c}\mu_{\operatorname{lin}} \\
\left(\mathrm{cm}^{2} / \mathrm{Vs}\right)\end{array}$ & $\begin{array}{c}\mu_{\mathrm{sat}} \\
\left(\mathrm{cm}^{2} / \mathrm{Vs}\right)\end{array}$ & $V_{\text {th }}(V)$ & $\Delta \mathbf{V}_{\text {th }}(\mathbf{V})$ & $\mathbf{I}_{\text {on }} / \mathbf{I}_{\text {off }}$ & $\mathrm{SS}(\mathrm{V} / \mathrm{dec})$ \\
\hline Non-treated & $0.07 \pm 0.02$ & $0.09 \pm 0.02$ & $-20.8 \pm 2.5$ & $9.5 \pm 1.1$ & $1.2 \times 10^{6}$ & $7.5 \pm 1.5$ \\
\hline 1-F & $0.09 \pm 0.01$ & $0.09 \pm 0.01$ & $-7.8 \pm 0.7$ & $5.4 \pm 0.8$ & $1.6 \times 10^{6}$ & $4.7 \pm 0.1$ \\
\hline $2-F$ & $0.07 \pm 0.02$ & $0.08 \pm 0.01$ & $-7.8 \pm 1.4$ & $6.3 \pm 1.4$ & $1.6 \times 10^{6}$ & $5.3 \pm 0.2$ \\
\hline $3-\mathrm{F}$ & $0.09 \pm 0.01$ & $0.12 \pm 0.03$ & $-8.2 \pm 1.1$ & $4.3 \pm 1.9$ & $2.2 \times 10^{6}$ & $4.8 \pm 0.1$ \\
\hline
\end{tabular}

Finally, we tried to understand more in detail the active layer formation on various surfaces. For that, very thin layer of active layer $(3 \mathrm{~nm})$ was deposited. AFM studies on these films are presented in Figure 7. DNTT forms large dispersed grains during initial growth. Here the sizes of these grains on the bare oxide and treated surfaces are similar being in the order of $500 \mathrm{~nm}$ large and $30 \mathrm{~nm}$ high. As confirmed earlier in surface energy analysis, comparable 
surface energies do not alter film formation of the active layer, consequently no drastic effect on charge transport, explaining similar OFET mobilities we achieved for these devices.

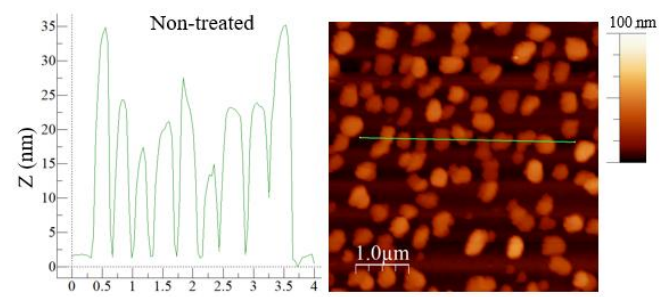

$\mathrm{X}(\mu \mathrm{m})$

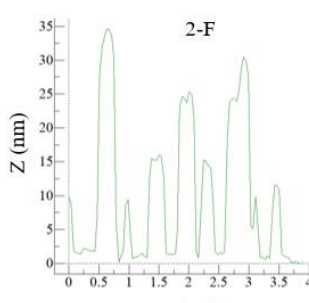

$\mathrm{X}(\mu \mathrm{m})$

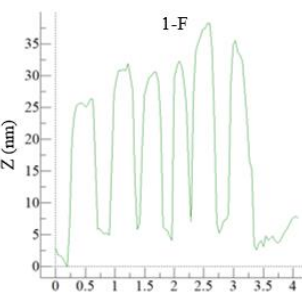

$\mathrm{X}(\mu \mathrm{m})$



$\frac{1.0 \mu m}{100}=$

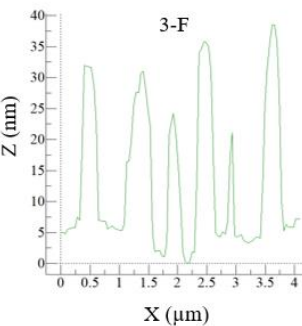

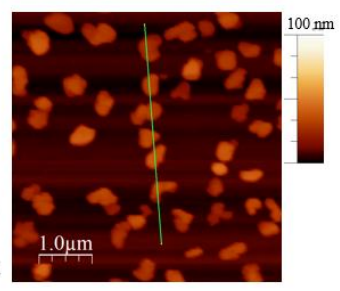

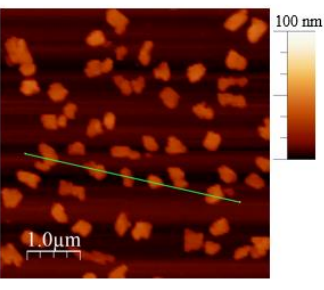

Figure 7. Atomic force microscopy (AFM) height images of very thin layer (3 nm) of DNTT actvie layer on bare $\mathrm{SiO}_{2}$ (nontreated) and treated surfaces. Line profiles in random direction are provided on the left of each image.

\section{Conclusions}

In summary, we report $\mathrm{SiO}_{2}$ dielectric surface modification with fluorinated phenylboronic acid molecules in the performance of OFETs. Although the surface energy does not show any noticeable change as evidenced by water contact angle measurements, we have obtained significant improvements in some device performance parameters, such as threshold voltage and subthreshold slope. As these parameters are correlated directly to the trap densities at the interface, grafting phenylboronic acid molecules on $\mathrm{SiO}_{2}$ is proven to be an efficient approach in passivating the $\mathrm{SiO}_{2}$ surface. Almost no effect on charge carrier mobilities and current on/off ratio were explained through the observation of the initial growth of active layer using AFM. Phenylboronic acid incorporated OFETs can find their potential in sensing applications, as they can be easily functionalized for various target molecules. This work can be a prelude for such studies.

\section{Acknowledgements}

This work is financially supported by Selcuk University Scientific Research Council (PN: 14101022). The authors thank to financial support of 2214-A International Doctoral Research Project (PN: 1059B141501270) and 2211-C Support through the Scientific and Technological Research Council of Turkey (TUBİTAK). 


\section{References}

${ }^{1}$ M. Kaltenbrunner, M.S. White, E.D. Głowacki, T. Sekitani, T. Someya, N.S. Sariciftci, and S. Bauer, Nat. Commun. 3, 770 (2012).

${ }^{2}$ H. Klauk, Chem. Soc. Rev. 39, 2643 (2010).

${ }^{3}$ D.G. Hall, Structure, Properties, and Preparation of Boronic Acid Derivatives. Overview of Their Reactions and Applications (2006).

${ }^{4}$ R. Nishiyabu, Y. Kubo, T.D. James, and J.S. Fossey, Chem. Commun. 47, 1124 (2011).

${ }^{5}$ K. Lacina, P. Skládal, and T.D. James, Chem. Cent. J. 8, 1 (2014).

${ }^{6}$ H. Chen, M. Lee, J. Lee, J.H. Kim, Y.S. Gal, Y.H. Hwang, W.G. An, and K. Koh, Sensors 7, 1480 (2007).

${ }^{7}$ S. Takahashi and J.I. Anzai, Langmuir 21, 5102 (2005).

${ }^{8}$ Y. Egawa, T. Seki, S. Takahashi, and J.I. Anzai, Mater. Sci. Eng. C 31, 1257 (2011).

${ }^{9}$ T. Minami, T. Minamiki, Y. Hashima, D. Yokoyama, T. Sekine, K. Fukuda, D. Kumaki, and S. Tokito, Chem. Commun. 50, 15613 (2014).

${ }^{10}$ A. Matsumoto, N. Sato, H. Cabral, K. Kataoka, and Y. Miyahara, Procedia Eng. 5, 926 (2010).

11 T. Kajisa and T. Sakata, ChemElectroChem 1, 1647 (2014).

${ }^{12}$ A. Matsumoto, N. Sato, and Y. Miyahara, Curr. Appl. Phys. 9, e214 (2009).

${ }^{13}$ S. Casalini, F. Leonardi, T. Cramer, and F. Biscarini, Org. Electron. 14, 156 (2013).

14 T. Yamamoto and K. Takimiya, J. Am. Chem. Soc. 129, 2224 (2007).

${ }^{15}$ S. Shinamura, I. Osaka, E. Miyazaki, A. Nakao, M. Yamagishi, J. Takeya, and K.

Takimiya, J. Am. Chem. Soc. 133, 5024 (2011).

${ }^{16}$ H. Yagi, T. Miyazaki, Y. Tokumoto, Y. Aoki, M. Zenki, T. Zaima, S. Okita, T. Yamamoto, E. Miyazaki, K. Takimiya, and S. Hino, Chem. Phys. Lett. 563, 55 (2013).

${ }^{17}$ G. Houin, F. Duez, L. Garcia, E. Cantatore, L. Hirsch, D. Belot, C. Pellet, and M. Abbas, Flex. Print. Electron. 2, 45004 (2017).

${ }^{18}$ M. Devynck, P. Tardy, G. Wantz, Y. Nicolas, L. Vellutini, C. Labrugère, and L. Hirsch, Appl. Phys. Lett. 100, 053308 (2012).

${ }^{19}$ M. Aghamohammadi, R. Rödel, U. Zschieschang, C. Ocal, H. Boschker, R.T. Weitz, E. Barrena, and H. Klauk, ACS Appl. Mater. Interfaces 7, 22775 (2015).

${ }^{20}$ U. Kraft, M. Sejfić, M.J. Kang, K. Takimiya, T. Zaki, F. Letzkus, J.N. Burghartz, E. Weber, and H. Klauk, Adv. Mater. 27, 207 (2015). 
${ }^{21}$ C.A. Di, G. Yu, Y. Liu, Y. Guo, X. Sun, J. Zheng, Y. Wen, Y. Wang, W. Wu, and D. Zhu, Phys. Chem. Chem. Phys. 11, 7268 (2009).

${ }^{22}$ S.H. Kim, W.M. Yun, O. Kwon, K. Hong, C. Yang, W. Choi, and C.E. Park, J. Phys. D Appl. Phys. 43, 465102 (2010).

${ }^{23}$ S. Zhou, Q. Tang, H. Tian, and X. Zhao, ACS Appl. Mater. Interfaces 10, 15943 (2018). 